Japan. J. Math.

Vol. 29, No. 2, 2003

\title{
Weak Bernoullicity of random walk in random scenery
}

\author{
By F. den Hollander, M.S. Keane, J. Serafin* and J.E. Steif ${ }^{\dagger}$
}

(Received May 2, 2003)

\begin{abstract}
In this paper, we consider an arbitrary irreducible random walk and an arbitrary stationary and ergodic random scenery on $\mathbb{Z}^{d}$. We find conditions on their distributions such that the associated random walk in random scenery is or is not weak Bernoulli. Our results extend an earlier classification for the special case in which the individual scenery values are assumed to be independent and identically distributed random variables.
\end{abstract}

\section{Background and main theorems}

1.1. Background. For a fixed integer $d \geq 1$, let $X=\left(X_{n}\right)_{n \in \mathbb{Z}}$ be independent and identically distributed random variables taking values in $\mathbb{Z}^{d}$ according to a common distribution $m$ on $\mathbb{Z}^{d}$ satisfying

$(* 1) \quad m$ is irreducible, i.e., $\operatorname{supp}(m)=\{x: m(x)>0\}$ generates the group $\mathbb{Z}^{d}$.

Let $S=\left(S_{n}\right)_{n \in \mathbb{Z}}$ be the corresponding random walk on $\mathbb{Z}^{d}$, defined by

$$
S_{0}=0, \quad S_{n}-S_{n-1}=X_{n} \quad(n \in \mathbb{Z}) .
$$

Next, let $C=\left(C_{x}\right)_{x \in \mathbb{Z}^{d}}$ be a random scenery on $\mathbb{Z}^{d}$ taking values in a finite set $F$ according to a distribution $\mu$ on $F^{\mathbb{Z}^{d}}$ satisfying

$(* 2) \quad \mu$ is stationary with respect to translations in the group $\mathbb{Z}^{d}$;

$(* 3) \quad \mu$ is ergodic with respect to translations in $\Gamma_{m}$, the subgroup of $\mathbb{Z}^{d}$ generated by $\operatorname{supp}(m)-\operatorname{supp}(m)=\{x-y: m(x) m(y)>0\}$.

The random walk and the random scenery are assumed to be independent.

The joint process

$$
Y=\left(Y_{n}\right)_{n \in \mathbb{Z}} \text { with } Y_{n}=\left(X_{n}, C_{S_{n}}\right)
$$

\footnotetext{
* Research supported in part by KBN grant 2 PO3A 04622 (2002-2005).

† Research supported in part by a grant from the Swedish Natural Science Research Council and by NSF-grant DMS-0103841.
} 
is called the random walk in random scenery associated with $m$ and $\mu$. In ergodic theory, $Y$ is an example of what is called a skew product.

It is obvious that, because of $(* 2), Y$ is a stationary process. The role of $(* 3)$ is to make $Y$ non-periodic. Indeed, if $F=\{0,1\}, d=1, m$ is simple random walk (i.e., $m(x)=1 / 2$ for $x= \pm 1$ and zero otherwise), and $\mu$ gives weight $1 / 2$ to the alternating configuration $(\ldots, 0,1,0,1,0, \ldots)$ and its shift, then the second component of $Y$ also gives weight $1 / 2$ to this configuration and its shift. The point here is that, although $\mu$ is ergodic under the full group $\mathbb{Z}$, it is not ergodic under the subgroup $\Gamma_{m}=2 \mathbb{Z}$.

In this paper, we are interested in the mixing properties of $Y$. A basic fact about $Y$ is the following, proved by Meilijson [11]:

Assume $(* 1),(* 2)$ and $(* 3)$. Then $Y$ has a trivial right tail, i.e., all sets in the tail $\sigma$-field $\bigcap_{N>0} \sigma\left(\left(Y_{n}\right)_{n \geq N}\right)$ have probability 0 or 1 .

The intuition behind this result is that for large $n$ the distribution of $S_{n}$ is close to uniform on a large box in some shift of $\Gamma_{m}$, so that the walk sees an ergodic average of the scenery. In ergodic theory, within the class of stationary processes whose one-dimensional distribution has finite entropy, a process with a trivial right tail is called a $K$-automorphism.

In den Hollander and Steif [8], two further mixing properties of $Y$ were investigated, namely, Bernoulli and weak Bernoulli. For the latter, the following result was obtained in [8] Theorem 2.2:

Assume that the scenery values are independent and identically distributed random variables. Then $Y$ is weak Bernoulli if and only if $|Z|<\infty$ a.s., where

$$
Z=\left\{S_{n}: n>0\right\} \cap\left\{S_{n}: n \leq 0\right\}
$$

is the intersection of the supports of the future and the past of the random walk.

Here, the intuition is that $|Z|<\infty$ if and only if the past and the far away future of the random walk are supported by disjoint sets, so that the walk sees independent sceneries.

The goal of the present paper is to extend the latter result to a more general class of random sceneries. One trivial extension is to finite block codings of independent and identically distributed random variables, but we want to push much further. Throughout the sequel, the conditions $(* 1),(* 2)$ and $(* 3)$ are in force.

In addition to the grant support acknowledged on the title page, the first author thanks Keio University and the other three authors thank Eurandom for hospitality at various stages of the development of this article.

1.2. Coupling representation. Before we state our main theorems, we recall the definition of weak Bernoulli and its coupling representation. A stationary 
process $Y=\left(Y_{n}\right)_{n \in \mathbb{Z}}$ is called weak Bernoulli if its past and its far away future are asymptotically independent in the sense of total variation, i.e.,

$$
\lim _{N \rightarrow \infty}\left\|P_{(-\infty, 0] \cup(N, \infty)}-P_{(-\infty, 0]} \times P_{(N, \infty)}\right\|_{t v}=0
$$

where $P_{A}$ is the distribution of $Y$ restricted to $A \cap \mathbb{Z}$ and $\|\cdot\|_{t v}$ denotes the total variation norm. In the course of the paper, we will need the following characterization of the weak Bernoulli property given in Berbee [1] Theorem 4.4.7:

A stationary process $Y=\left(Y_{n}\right)_{n \in \mathbb{Z}}$ is weak Bernoulli if and only if there is a joint process $\left(Y_{n}^{\prime}, Y_{n}^{\prime \prime}\right)_{n \in \mathbb{Z}}$ such that:

(1) $\left(Y_{n}^{\prime}\right)_{n \in \mathbb{Z}}$ and $\left(Y_{n}^{\prime \prime}\right)_{n \in \mathbb{Z}}$ are equal to $\left(Y_{n}\right)_{n \in \mathbb{Z}}$ in distribution;

(2) $\left(Y_{n}^{\prime}\right)_{n \in \mathbb{Z}}$ and $\left(Y_{n}^{\prime \prime}\right)_{n \leq 0}$ are independent;

(3) a.s. there exists a (random) nonnegative integer $N$ such that $Y_{n}^{\prime}=Y_{n}^{\prime \prime}$ for $n>N$.

(The last condition is expressed by saying that the two copies of the process are "successfully coupled" after time $N$.)

We will also need a Cesaro-version of the weak Bernoulli property of a stationary process $Y$, which is slightly weaker and is defined as follows:

$$
\lim _{N \rightarrow \infty}\left\|\frac{1}{N} \sum_{M=0}^{N-1} P_{M}-P_{(-\infty, 0]} \times P_{(0, \infty)}\right\|_{t v}=0 .
$$

Here, $P_{M}$ is the distribution of $\left(\tilde{Y}_{n}\right)_{n \in \mathbb{Z}}$ with $\tilde{Y}_{n}=Y_{n}$ for $n \leq 0$ and $\tilde{Y}_{n}=Y_{n+M}$ for $n>0$. As shown in Berbee [1] Theorem 4.4.9, this property has the same coupling representation as above, except that condition (3) is replaced by

$\left(3^{\prime}\right)$ a.s. there exist (random) nonnegative integers $M_{1}, M_{2}$ such that $Y_{n+M_{1}}^{\prime}=$ $Y_{n+M_{2}}^{\prime \prime}$ for $n>0$.

(The last condition is expressed by saying that the two copies of the process are "successfully shift-coupled" after time $M_{1} \vee M_{2}$.)

1.3. Not weak Bernoulli. Our first main theorem is general and reads:

Theorem 1. If $\mu$ has no atoms and $|Z|=\infty$ a.s., then $Y$ is not weak Bernoulli.

Remarks. (a) By exchangeability of $X$, the event $|Z|=\infty$ has probability 0 or 1 (see [8] Section 2.1).

(b) It is well-known that for simple random walk (i.e., $m(x)=1 / 2 d$ for $\|x\|=1$ and zero otherwise) $|Z|=\infty$ a.s. if and only if $1 \leq d \leq 4$ (see e.g. Lawler [9] Section 3). Further examples can be found in [8] Theorem 2.5, Theorem 5.3 and Corollary 5.6 (which include all $m$ in $1 \leq d \leq 4$ with zero mean and finite variance). 
(c) Theorem 1 is optimal. Indeed, under conditions $(* 1),(* 2)$ and $(* 3)$, if $\mu$ has atoms, then it lives on a single periodic configuration and its shifts, such that the periods of the scenery and the walk are different. Consequently, $Y$ has a nonperiodic renewal type structure, from which the weak Bernoulli property easily follows. Note that in this case the weak Bernoulli property holds even when the random walk is recurrent (which allows the full scenery $C$ to be recovered from the past of $Y$ ).

1.4. One dimension. Theorem 1 raises the question whether there are mild conditions on $\mu$ such that $Y$ is weak Bernoulli when $|Z|<\infty$ a.s. Our second main theorem provides a class of random walks for which this is not the case.

Theorem 2. Let $d=1$. Assume that $m$ is right-continuous (i.e., $m(x)=0$ for $x>1$ ), has positive drift (i.e., $\left.\sum_{x} x m(x)>0\right)$, and $\Gamma_{m}=\mathbb{Z}$. Then $Y$ is weak Bernoulli if and only if $\mu$ is Cesaro weak Bernoulli.

REMARKs. (a) The positive drift of $m$ implies that $|Z|<\infty$ a.s.

(b) At the end of Section 3 we will see that if $\mu$ has no atoms and has zero entropy, then it is not Cesaro weak Bernoulli. (See Walters [13] Chapter 4 for the definition of entropy.)

(c) Theorem 2 may be extended to higher dimensions as follows. Let $C_{x}=$ $\left(C_{x_{1}}^{1}, \ldots, C_{x_{d}}^{d}\right)$ with $\left(C_{x_{i}}^{i}\right)_{x_{i} \in \mathbb{Z}}$ independent one-dimensional random sceneries with distributions $\mu^{i}$. Put $m_{i}(y)=\sum_{x: x_{i}=y} m(x), y \in \mathbb{Z}$, and assume that, for each $i$, $m_{i}$ is right-continuous, has positive drift, and $\Gamma_{m_{i}}=\mathbb{Z}$. Then $Y$ is weak Bernoulli if and only if $\mu^{i}$ is Cesaro weak Bernoulli for all $i$.

1.5. Weak Bernoulli. Theorem 1 says that if $|Z|=\infty$ a.s., then $\mu$ having no atoms is enough to make $Y$ not weak Bernoulli. We would like to prove that, conversely, if $|Z|<\infty$ a.s., then $Y$ is weak Bernoulli for "most" random fields $\mu$. The reason why this is difficult is that it comes down to showing that the sceneries on two random infinite sets, namely, $\left\{S_{n}: n \leq 0\right\}$ and $\left\{S_{n}: n \geq N\right\}$, are close to independent when $N$ is large (since then these sets are far away from each other). Unfortunately, there are very few tools available to do this. Below we will employ one such tool, based on disagreement percolation.

In Theorem 2, the two infinite sets are simple, namely, two infinite rays in opposite directions. This allows us to directly relate the mixing properties of $C$ and $Y$. In more general situations we need more structure. In Theorems 3 and 4 below we will obtain a classification for Markov random fields, both in the "low noise" and the "high noise" regime (see Georgii [4] Section 8.2 for the definition of a Markov random field).

A. Low noise regime. Our third main theorem uses the notion of disagreement percolation for two independent realizations of the random scenery. For 
$x \in \mathbb{Z}^{d}$, let

$$
E_{\neq}(x)=\left\{\left(c^{\prime}, c^{\prime \prime}\right) \in F^{\mathbb{Z}^{d}} \times F^{\mathbb{Z}^{d}}: \exists \pi: 0 \longleftrightarrow x \text { such that } c^{\prime}(y) \neq c^{\prime \prime}(y) \forall y \in \pi\right\}
$$

be the set of pairs of sceneries such that there is a nearest-neighbor path between 0 and $x$ (including 0 and $x$ itself) on which the sceneries disagree everywhere, and put

$$
\phi_{\mu}(x)=(\mu \times \mu)\left(E_{\neq}(x)\right) .
$$

Let

$$
S_{+}=\left\{S_{n}: n>0\right\} \text { and } S_{-}=\left\{S_{n}: n \leq 0\right\}
$$

denote the support of the future, respectively, the past of the random walk, and put

$$
\Phi_{\mu}=\sum_{\substack{x \in S_{+} \\ y \in S_{-}}} \phi_{\mu}(x-y) .
$$

Theorem 3. Assume that $\mu$ is Markov, $\sum_{x} \phi_{\mu}(x)<\infty$ and $\Phi_{\mu}<\infty$ a.s. Then $Y$ is weak Bernoulli.

Remarks. (a) Again, by exchangeability of $X$, the event $\Phi_{\mu}<\infty$ has probability 0 or 1 .

(b) Unless $\mu$ lives on a single constant configuration, in which case $\phi_{\mu} \equiv 0$, we have $\phi_{\mu}(0)>0$ and so $\Phi_{\mu}<\infty$ implies that $|Z|<\infty$.

B. High noise regime. Our fourth main theorem uses standard percolation to bound disagreement percolation. For $\mu$ Markov, define

$$
p(\mu)=\max _{\eta, \eta^{\prime} \in F^{\partial\{0\}}}\left\|\mu\left(C_{0} \in \cdot|C|_{\partial\{0\}}=\eta\right)-\mu\left(C_{0} \in \cdot|C|_{\partial\{0\}}=\eta^{\prime}\right)\right\|_{t v}
$$

with $\partial\{0\}=\{x:\|x\|=1\}$. (Throughout the paper, $\|x\|=\sum_{1 \leq i \leq d}\left|x_{i}\right|$ denotes the $\ell_{1}$-norm of $x \in \mathbb{Z}^{d}$.) A small $p(\mu)$ corresponds to the scenery values being close to independent. In particular, all $\mu$ that are "Gibbs measures with a summable potential" have a corresponding $p(\mu)$ that tends to zero as the "temperature" tends to infinity (see Georgii [4] Chapter 1 for the definition of a Gibbs measure).

For $x \in \mathbb{Z}^{d}$, let

$$
E_{1}(x)=\left\{d \in\{0,1\}^{\mathbb{Z}^{d}}: \exists \pi: 0 \longleftrightarrow x \text { such that } d(y)=1 \forall y \in \pi\right\}
$$

and put

$$
\tilde{\phi}_{\mu}(x)=P_{p(\mu)}\left(E_{1}(x)\right)
$$

with $P_{p}$ the probability measure for standard site percolation with parameter $p \in$ $[0,1]$, i.e., each site $x$ is open $(d(x)=1)$ with probability $p$ and closed $(d(x)=0)$ 
with probability $1-p$ independently of the other sites (see Grimmett [7] Section 1.6). Let

$$
\tilde{\Phi}_{\mu}=\sum_{\substack{x \in S_{+} \\ y \in S_{-}}} \tilde{\phi}_{\mu}(x-y) .
$$

Theorem 4. Assume that $\mu$ is Markov, $\sum_{x} \tilde{\phi}_{\mu}(x)<\infty$ and $\tilde{\Phi}_{\mu}<\infty$ a.s. Then $Y$ is weak Bernoulli.

C. Random walk classes. Our fifth main theorem provides conditions on $m$ under which $\Phi_{\mu}<\infty$ a.s. or $\tilde{\Phi}_{\mu}<\infty$ a.s.

Theorem 5. Let $f: \mathbb{Z}^{d} \rightarrow[0,1]$ and $F=\sum_{x \in S_{+}, y \in S_{-}} f(x-y)$. Then $E(F)<\infty$, with $E$ expectation over $S$, in either of the following cases:

(i) $d \geq 5, \sum_{x} f(x)<\infty$;

(ii) $1 \leq d \leq 4, \sum_{x}\|x\| f(x)<\infty, \sum_{x} x m(x) \neq 0, \sum_{x}\|x\|^{6} m(x)<\infty$.

In Burton and Steif [3] Proposition 2.4, it is shown that

$$
\phi_{\mu}(x) \leq C e^{-c\|x\|} \text { for } x \in \mathbb{Z}^{d} \text { and some } c>0, C<\infty,
$$

when $\mu$ is the plus-phase or the minus-phase of the nearest-neighbor ferromagnetic Ising model $(F=\{-1,+1\})$ on $\mathbb{Z}^{d}, d \geq 2$, at sufficiently low temperature. Via Theorems 3 and 5, this yields a class of "low noise" random sceneries and a class of random walks with $|Z|<\infty$ a.s. for which $Y$ is weak Bernoulli. In $d=1$, for this model the same bound on $\phi_{\mu}$ holds at any temperature. Two further examples of a $\mu$ for which the same bound on $\phi_{\mu}$ holds are given in [3] Propositions 3.1 and 4.1, both based on a nearest-neighbor subshift of finite type.

REMARKs. (a) It is not hard to extend the argument in [3] to cover the nearest-neighbor ferromagnetic Potts model $(F=\{1, \ldots, q\}, q \in \mathbb{N})$ on $\mathbb{Z}^{d}, d \geq 2$. Thus, the above bound on $\phi_{\mu}$ also holds for the $q$ pure phases of the Potts model at sufficiently low temperature.

(b) The restriction to sufficiently low temperature is necessary to get control over the disagreement percolation under $\mu \times \mu$. As we will see in Section 4, disagreement percolation under $\mu \times \mu$ is a powerful tool, but it may seriously overestimate the total variation norm.

It is well known (see Grimmett [7] Theorem 5.4) that

$$
\tilde{\phi}_{\mu}(x) \leq C e^{-c\|x\|} \text { for } x \in \mathbb{Z}^{d} \text { and some } c>0, C<\infty,
$$

when $0 \leq p(\mu)<p_{c}(d)$ with $p_{c}(d)$ the critical value for standard site percolation on $\mathbb{Z}^{d}$, which satisfies $p_{c}(1)=1$ and $p_{c}(d) \in(0,1), d \geq 2$. Via Theorems 4 and 5 , this 
yields a class of "high noise" random sceneries and a class of random walks with $|Z|<\infty$ a.s. for which $Y$ is weak Bernoulli.

Concluding, the Markov assumption on $\mu$ in Theorems 3 and 4 is rather restrictive, but at the beginning of this subsection we have explained why it is hard to go further. We expect that $Y$ is weak Bernoulli for all random sceneries that are stationary extremal Gibbs measures with a sufficiently tempered interaction potential at any non-critical temperature and all random walks with $|Z|<\infty$ a.s., but currently this seems beyond reach.

\section{Proof of Theorem 1}

We assume that $\mu$ has no atoms, $|Z|=\infty$ a.s. and that $Y$ is weak Bernoulli. We show that this leads to a contradiction. It was proved in [8] Lemma 3.2 that if $|Z|=\infty$ a.s., then a.s.

$$
\left|\left\{z \in \mathbb{Z}^{d}: z+B_{k} \subseteq Z\right\}\right|=\infty \quad \forall k \in \mathbb{N},
$$

where $B_{k}=[-k, k]^{d} \cap \mathbb{Z}^{d}$ is the box of size $k$ centered at the origin. We will need this fact as we go along.

According to the coupling representation in Section 1.2, since $Y$ is weak Bernoulli, we have copies

$$
\left(X_{n}^{\prime}, C_{S_{n}^{\prime}}^{\prime}\right)_{n \in \mathbb{Z}} \quad \text { and } \quad\left(X_{n}^{\prime \prime}, C_{S_{n}^{\prime \prime}}^{\prime \prime}\right)_{n \in \mathbb{Z}}
$$

of the process $\left(X_{n}, C_{S_{n}}\right)_{n \in \mathbb{Z}}$ satisfying conditions (1), (2) and (3). Let $\widehat{P}, \widehat{E}$ denote probability and expectation under this coupling. If $N$ is as in condition (3), then after time $N$ both walks take the same steps and see the same scenery values. Therefore we have

$$
\widehat{P}\left(\bigcup_{w \in \Gamma_{m}} \bigcup_{N \in \mathbb{N}_{0}} \bigcap_{n>N}\left\{S_{n}^{\prime}+w=S_{n}^{\prime \prime}, C_{S_{n}^{\prime}}^{\prime}=C_{S_{n}^{\prime \prime}}^{\prime \prime}\right\}\right)=1 .
$$

Put

$$
\tilde{Z}=\left\{S_{n}^{\prime}: n>0\right\} \cap\left\{S_{n}^{\prime \prime}: n \leq 0\right\} .
$$

By conditions (1) and (2), $\tilde{Z}$ is equal to $Z$ in distribution. Hence, by $(\triangle)$, there is a sequence $\left(z_{k}\right)_{k \in \mathbb{N}}$ in $\mathbb{Z}^{d}$ such that $z_{k}+B_{2 k} \subseteq \tilde{Z}$. In order to properly define these random variables, we consider an arbitrary fixed ordering of $\mathbb{Z}^{d}$ and, for each $k \in \mathbb{N}$, we let $z_{k}$ be the smallest element in $\mathbb{Z}^{d}$ (with respect to this ordering) such that $z_{k}+B_{2 k} \subseteq \tilde{Z}$. Observe that $\left(z_{k}\right)_{k \in \mathbb{N}}$ is measurable with respect to the pair $\left(S_{n}^{\prime}\right)_{n>0},\left(S_{n}^{\prime \prime}\right)_{n \leq 0}$.

Let $\left.C\right|_{A}$ denote the scenery $C$ restricted to the set $A$. Since $\left(S_{n}^{\prime}\right)_{n>0}$ visits all of the box $z_{k}+B_{k},\left.C^{\prime}\right|_{z_{k}+B_{k}}$ is defined. If $k \geq\|w\|$, then $z_{k}+B_{k}+w \subseteq z_{k}+B_{2 k} \subseteq \tilde{Z}$, 
and so also $\left.C^{\prime \prime}\right|_{z_{k}+B_{k}+w}$ is defined. From this observation and the above coupling statement, it follows that

$$
\widehat{P}\left(\bigcup_{w \in \Gamma_{m}} \bigcup_{K \geq\|w\| k \geq K} \bigcap_{k}\left\{\left.C^{\prime}\right|_{z_{k}+B_{k}}=\left.C^{\prime \prime}\right|_{z_{k}+B_{k}+w}\right\}\right)=1 .
$$

(Note that, given a realization of the process $Y$, it only makes sense to talk about the scenery values at the sites that are visited by the walk.) We will show that, for all $w \in \mathbb{Z}^{d}$

$$
\widehat{P}\left(\bigcup_{K \geq\|w\| k \geq K} \bigcap_{k}\left\{\left.C^{\prime}\right|_{z_{k}+B_{k}}=\left.C^{\prime \prime}\right|_{z_{k}+B_{k}+w}\right\}\right)=0
$$

which yields the desired contradiction. The latter probability is equal to

$$
\widehat{E}\left[\widehat{P}\left(\bigcup_{K \geq\|w\| k \geq K} \bigcap_{k}\left\{\left.C^{\prime}\right|_{z_{k}+B_{k}}=\left.C^{\prime \prime}\right|_{z_{k}+B_{k}+w}\right\} \mid \tilde{Z}\right)\right]
$$

and so it suffices to show that

$$
\widehat{P}\left(\bigcup_{K \geq\|w\|} \bigcap_{k \geq K}\left\{\left.C^{\prime}\right|_{z_{k}+B_{k}}=\left.C^{\prime \prime}\right|_{z_{k}+B_{k}+w}\right\} \mid \tilde{Z}\right)=0 \quad \widehat{P} \text {-a.s. }
$$

By conditions (1) and (2), given any realization of $\left(X_{n}^{\prime}\right)_{n>0}$ and $\left(X_{n}^{\prime \prime}\right)_{n \leq 0}$ (and hence of $\tilde{Z}$ and $\left(z_{k}\right)$, which are measurable with respect to this pair of sequences), we have that

$$
\left(C_{x}^{\prime}\right)_{x \in \tilde{Z}} \text { and }\left(C_{x}^{\prime \prime}\right)_{x \in \tilde{Z}}
$$

are independent and are distributed according to $\mu$ restricted to $\tilde{Z}$. It follows that, to verify the statement in the last display, it suffices to show that for all $w$ and $\left(z_{k}\right)$,

$$
(\mu \times \mu)\left(\bigcup_{K \geq\|w\| k \geq K} \bigcap_{k}\left\{\left.C^{\prime}\right|_{B_{k}+z_{k}}=\left.C^{\prime \prime}\right|_{B_{k}+z_{k}+w}\right\}\right)=0 .
$$

By Fatou's lemma, it in turn suffices to show that for all $w$ and $\left(z_{k}\right)$,

$$
\liminf _{k \rightarrow \infty}(\mu \times \mu)\left(\left\{\left.C^{\prime}\right|_{B_{k}+z_{k}}=\left.C^{\prime \prime}\right|_{B_{k}+z_{k}+w}\right\}\right)=0 .
$$

By the stationarity of $\mu$, it in turn suffices to show that

$$
\liminf _{k \rightarrow \infty}(\mu \times \mu)\left(E_{k}\right)=0
$$


where

$$
E_{k}=\left\{\left(c^{\prime}, c^{\prime \prime}\right):\left.c^{\prime}\right|_{B_{k}}=\left.c^{\prime \prime}\right|_{B_{k}}\right\} .
$$

Since $\mu$ has no atoms, if $\tilde{c}$ is any fixed scenery, then

$$
\mu\left(\left\{c:\left.c\right|_{B_{k}}=\left.\tilde{c}\right|_{B_{k}}\right\}\right) \rightarrow 0 \text { as } k \rightarrow \infty
$$

We even have

$$
\sup _{\bar{c}} \mu\left(\left\{c:\left.c\right|_{B_{k}}=\left.\tilde{c}\right|_{B_{k}}\right\}\right) \rightarrow 0 \text { as } k \rightarrow \infty .
$$

Indeed, if the latter would fail, then there would exist an $\epsilon>0$, a sequence $k_{i}, i \in \mathbb{N}$, of positive integers tending to infinity, and a sequence $\tilde{c}_{i}, i \in \mathbb{N}$, of sceneries such that

$$
\mu\left(\left\{c:\left.c\right|_{B_{k_{i}}}=\left.\tilde{c}_{i}\right|_{B_{k_{i}}}\right\}\right)>\epsilon \text { for } i \in \mathbb{N} .
$$

Picking a subsequence if necessary (via a diagonal argument), we would be able to produce a sequence $\tilde{c}_{i}, i \in \mathbb{N}$, of sceneries converging, on any finite block, to some scenery $\tilde{c}$ for which $\mu(\tilde{c}) \geq \epsilon$. But this would contradict the assumption that $\mu$ has no atoms.

Thus, abbreviating $\epsilon_{k}(\bar{c})=\mu\left(\left\{c:\left.c\right|_{B_{k}}=\bar{c}\right\}\right), \bar{c} \in F^{B_{k}}$, we have that for any $\epsilon>0$ there exists a positive integer $k_{\epsilon}$ such that

$$
\sup _{\bar{c} \in F^{B_{k}}} \epsilon_{k}(\bar{c}) \leq \epsilon \quad \text { for } k \geq k_{\epsilon}
$$

Now write

$$
\begin{aligned}
E_{k} & =\left\{\left(c^{\prime}, c^{\prime \prime}\right):\left.c^{\prime}\right|_{B_{k}}=\left.c^{\prime \prime}\right|_{B_{k}}\right\} \\
& =\bigcup_{\bar{c} \in F^{B_{k}}}\left\{\left(c^{\prime}, c^{\prime \prime}\right):\left.c^{\prime}\right|_{B_{k}}=\left.c^{\prime \prime}\right|_{B_{k}}=\bar{c}\right\} \\
& =\bigcup_{\bar{c} \in F^{B_{k}}}\left[\left\{\left(c^{\prime}, \cdot\right):\left.c^{\prime}\right|_{B_{k}}=\bar{c}\right\} \bigcap\left\{\left(\cdot, c^{\prime \prime}\right):\left.c^{\prime \prime}\right|_{B_{k}}=\bar{c}\right\}\right]
\end{aligned}
$$

and estimate

$$
(\mu \times \mu)\left(E_{k}\right) \leq \sum_{\bar{c} \in F^{B_{k}}} \epsilon_{k}(\bar{c})^{2} \leq \epsilon \sum_{\bar{c} \in F^{B_{k}}} \epsilon_{k}(\bar{c})=\epsilon \text { for } k \geq k_{\epsilon} .
$$

Hence $\lim \sup _{k \rightarrow \infty}(\mu \times \mu)\left(E_{k}\right) \leq \epsilon$. Let $\epsilon \downarrow 0$ to complete the proof. 


\section{Proof of Theorem 2}

if: Suppose that $\mu$ is Cesaro weak Bernoulli. Then there is a coupling of two copies of the random scenery $\tilde{C}^{\prime}=\left(\tilde{C}_{x}^{\prime}\right)_{x \in \mathbb{Z}}$ and $\tilde{C}^{\prime \prime}=\left(\tilde{C}_{x}^{\prime \prime}\right)_{x \in \mathbb{Z}}$ such that $\left(\tilde{C}_{x}^{\prime}\right)_{x \in \mathbb{Z}}$ and $\left(\tilde{C}_{x}^{\prime \prime}\right)_{x \leq 0}$ are independent and a.s. there exist (random) nonnegative integers $M_{1}, M_{2}$ such that

$$
\tilde{C}_{x+M_{1}}^{\prime}=\tilde{C}_{x+M_{2}}^{\prime \prime} \text { for } x>0 .
$$

(Note that, while $M_{1}$ and $M_{2}$ are only defined up to a constant, $M_{1}-M_{2}$ is uniquely defined.) Next, let $X_{-}^{\prime}=\left(X_{n}^{\prime}\right)_{n \leq 0}$ and $X_{-}^{\prime \prime}=\left(X_{n}^{\prime \prime}\right)_{n \leq 0}$ be two independent copies of the random walk steps for nonpositive times, which we also assume to be independent of $\tilde{C}^{\prime}$ and $\tilde{C}^{\prime \prime}$. Let $\left(S_{n}^{\prime \prime}\right)$ be the random walk corresponding to $\left(X_{n}^{\prime \prime}\right)$, and put $R=\max \left\{S_{n}^{\prime \prime}: n \leq 0\right\} \geq 0$, which is finite by our assumption that the random walk has positive drift. Now define

$$
C_{x}^{\prime}=\tilde{C}_{x-R}^{\prime} \text { and } C_{x}^{\prime \prime}=\tilde{C}_{x-R}^{\prime \prime} \text { for } x \in \mathbb{Z} .
$$

Observe that $\left(C_{x}^{\prime}\right)_{x \in \mathbb{Z}}$ and $\left(C_{x}^{\prime \prime}\right)_{x \leq R}$ are independent.

Next, for any $k$ let $\left({ }^{k} X_{n}^{\prime}\right)_{n>0}$ and $\left({ }^{k} X_{n}^{\prime \prime}\right)_{n>0}$ be two copies of the random walk steps for positive times such that a.s. there exists a (random) nonnegative integer $N$ such that

$$
{ }^{k} S_{n}^{\prime}={ }^{k} S_{n}^{\prime \prime}+k \text { for } n>N
$$

This coupling exists by Georgii [5] Lemma 2.2, because of our assumption that $\Gamma_{m}=\mathbb{Z}$. We assume that these couplings are independent for different $k$ and also independent of all the other random variables defined earlier.

We now define processes $Y^{\prime}$ and $Y^{\prime \prime}$ in terms of the above random variables as follows. Let the first coordinate of $Y_{n}^{\prime}$, which we call $\tilde{X}_{n}^{\prime}$, be

$$
\begin{aligned}
\tilde{X}_{n}^{\prime} & =X_{n}^{\prime} & \text { for } n \leq 0 \\
& =M_{1}-M_{2} X_{n}^{\prime} & \text { for } n>0
\end{aligned}
$$

and let the second coordinate of $Y_{n}^{\prime}$ be $C_{\tilde{S}_{n}^{\prime}}^{\prime}$, where $\left(\tilde{S}_{n}^{\prime}\right)$ is the random walk corresponding to $\left(\tilde{X}_{n}^{\prime}\right)$. Analogously, let the first coordinate of $Y_{n}^{\prime \prime}$, which we call $\tilde{X}_{n}^{\prime \prime}$, be

$$
\begin{aligned}
\tilde{X}_{n}^{\prime \prime} & =X_{n}^{\prime \prime} & \text { for } n \leq 0, \\
& =M_{1}-M_{2} X_{n}^{\prime \prime} & \text { for } n>0,
\end{aligned}
$$

and let the second coordinate of $Y_{n}^{\prime \prime}$ be $C_{\tilde{S}_{n}^{\prime \prime}}^{\prime \prime}$, where $\left(\tilde{S}_{n}^{\prime \prime}\right)$ is the random walk corresponding to $\left(\tilde{X}_{n}^{\prime \prime}\right)$. From the construction it follows (after some reflection) that $Y^{\prime}$ and $Y^{\prime \prime}$ satisfy properties (1), (2) and (3). 
only if: Suppose that $Y$ is weak Bernoulli. Then, as in the proof in Section 2, we have copies

$$
\left(X_{n}^{\prime}, C_{S_{n}^{\prime}}^{\prime}\right)_{n \in \mathbb{Z}} \text { and }\left(X_{n}^{\prime \prime}, C_{S_{n}^{\prime \prime}}^{\prime \prime}\right)_{n \in \mathbb{Z}}
$$

of the process $\left(X_{n}, C_{S_{n}}\right)_{n \in \mathbb{Z}}$ satisfying conditions (1), (2) and (3), the latter stating that a.s.

$$
S_{n}^{\prime}+w=S_{n}^{\prime \prime} \text { and } C_{S_{n}^{\prime}}^{\prime}=C_{S_{n}^{\prime \prime}}^{\prime \prime} \text { for } n>N
$$

for a (random) $w \in \mathbb{Z}$ and a (random) nonnegative integer $N$. Since $S_{(N, \infty)}^{\prime \prime} \supset$ $\left(S_{N}^{\prime \prime}, \infty\right)$ a.s. by our assumption that the random walk is right-continuous and has positive drift, it follows that a.s.

$$
C_{x-w}^{\prime}=C_{x}^{\prime \prime} \quad \text { for } x>S_{N}^{\prime \prime}
$$

Moreover, since $S_{\mathbb{Z}}^{\prime}=\mathbb{Z}$ and $S_{(-\infty, 0]}^{\prime \prime} \supset(-\infty, 0]$ a.s., it follows from conditions (1) and (2) that

$$
\left(C_{x}^{\prime}\right)_{x \in \mathbb{Z}} \text { and }\left(C_{x}^{\prime \prime}\right)_{x \leq 0}
$$

are independent. Thus, conditions (1), (2) and $\left(3^{\prime}\right)$ hold for $\left(C_{x}^{\prime}\right)_{x \in \mathbb{Z}}$ and $\left(C_{x}^{\prime \prime}\right)_{x \in \mathbb{Z}}$ with $M_{1}=M_{2}-w$ and $M_{2}=S_{N}^{\prime \prime}$ (pick $M_{2} \geq w$ ). Hence $\mu$ is Cesaro weak Bernoulli.

Note that the "if" direction does not require the right-continuity of the random walk, while the "only if" direction does not require that $\Gamma_{m}=\mathbb{Z}$.

We close this section by proving the claim made in Remark (b) below Theorem 2. Suppose that $\mu$ is Cesaro weak Bernoulli. Then, as above, there exist random sceneries

$$
\left(C_{x}^{\prime}\right)_{x \in \mathbb{Z}} \text { and }\left(C_{x}^{\prime \prime}\right)_{x \in \mathbb{Z}}
$$

such that conditions (1), (2) and $\left(3^{\prime}\right)$ hold, the latter stating that a.s.

$$
C_{x-w}^{\prime}=C_{x}^{\prime \prime} \text { for } x>\hat{N}
$$

for a (random) $w \in \Gamma_{m}=\mathbb{Z}$ and a (random) nonnegative integer $\hat{N}(\geq w)$. Now, if $\mu$ has zero entropy, then $\left(C_{x}^{\prime \prime}\right)_{x>0}$ can be retrieved from $\left(C_{x}^{\prime \prime}\right)_{x \leq 0}$ a.s. Thus, we have two independent copies $\left(C_{x}^{\prime}\right)_{x \in \mathbb{Z}}$ and $\left(C_{x}^{\prime \prime}\right)_{x \in \mathbb{Z}}$ of the random scenery such that a.s.

$$
C_{x-w}^{\prime}=C_{x}^{\prime \prime} \text { for } x>\hat{N} .
$$

This in turn implies that

$$
(\mu \times \mu)\left(\bigcup_{w \in \Gamma_{m}} \bigcup_{\hat{N} \in \mathbb{N}_{0}}\left\{C_{x-w}^{\prime}=C_{x}^{\prime \prime} \forall x>\hat{N}\right\}\right)=1
$$

But, by the argument given at the end of Section 2, the latter is impossible when $\mu$ has no atoms. 


\section{Proof of Theorem 3}

We assume that $\Phi_{\mu}<\infty$ a.s. and construct a coupling satisfying conditions (1), (2) and (3). The key to the construction is the following property. Let

$$
S_{-}=\left\{S_{n}: n \leq 0\right\} \quad \text { and } \quad S_{+}^{N}=\left\{S_{n}: n \geq N\right\}, \quad N \in \mathbb{N},
$$

denote the support of the past, respectively, the future beyond time $N$ of the random walk. We say that the random scenery is weak Bernoulli along the random walk if

$$
\lim _{N \rightarrow \infty}\left\|\left.\mu\right|_{S_{-} \cup S_{+}^{N}}-\left.\mu\right|_{S_{-}} \times\left.\mu\right|_{S_{+}^{N}}\right\|_{t v}=0 \text { for a.s. all } S,
$$

where $\left.\mu\right|_{A}$ denotes the restriction of $\mu$ to $A$. We first construct the coupling (with properties (1), (2) and (3)) assuming (§). After that we show that $\Phi_{\mu}<\infty$ a.s. implies $(\S)$.

STEP 1. (§) implies that $Y$ is weak Bernoulli.

Proof. Let

$$
X^{\prime}=\left(X_{n}^{\prime}\right)_{n \in \mathbb{Z}}, C^{\prime}=\left(C_{x}^{\prime}\right)_{x \in \mathbb{Z}^{d}}, X^{\prime \prime}=\left(X_{n}^{\prime \prime}\right)_{n \in \mathbb{Z}}, C^{\prime \prime}=\left(C_{x}^{\prime \prime}\right)_{x \in \mathbb{Z}^{d}},
$$

be independent copies of the random walk and the random scenery. Let $S^{\prime}=$ $\left(S_{n}^{\prime}\right)_{n \in \mathbb{Z}}$ and $S^{\prime \prime}=\left(S_{n}^{\prime \prime}\right)_{n \in \mathbb{Z}}$ be the associated random walks. Define

$$
\begin{array}{cc}
Y_{n}^{\prime}=\left(X_{n}^{\prime}, C_{S_{n}^{\prime}}^{\prime}\right), \quad n \in \mathbb{Z}, \\
Y_{n}^{\prime \prime}=\left(X_{n}^{\prime \prime}, C_{S_{n}^{\prime \prime}}^{\prime \prime}\right), \quad n \leq 0 .
\end{array}
$$

For $n>0$, let the first component of $Y_{n}^{\prime \prime}$ be $X_{n}^{\prime}$. Then, clearly, condition (2) and most of condition (1) hold. We now need to define the second component of $Y_{n}^{\prime \prime}$ for $n>0$ in such a way that condition (3) and the rest of condition (1) hold.

By $(\S)$, we have that a.s.

$$
\lim _{N \rightarrow \infty}\left\|\left.\mu\right|_{S_{-}^{\prime \prime} \cup S_{+}^{\prime N}}-\left.\mu\right|_{S_{-}^{\prime \prime}} \times\left.\mu\right|_{S_{+}^{\prime N}}\right\| \|_{t v}=0 .
$$

On the set of measure zero where the latter fails to hold, let the second coordinate of $Y_{n}^{\prime \prime}$ for $n>0$ be some fixed element $f_{0} \in F$. On the set of measure one where it holds, define the second coordinate of $Y_{n}^{\prime \prime}$ for $n>0$ as follows. By a trivial extension of Berbee [1] Equation (4.4.2) to the present nonstationary setting, on this latter set we have a.s.

$$
\lim _{N \rightarrow \infty}\left\|\left.\mu\right|_{S_{+}^{\prime N} \mid S_{-}^{\prime \prime}}-\left.\mu\right|_{S_{+}^{\prime N}}\right\|_{t v}=0
$$


where $\left.\mu\right|_{A \mid B}$ denotes the restriction of $\mu$ to $A$ conditional on the scenery in $B$. (Note that, even with the walk fixed, $\left\|\left.\mu\right|_{S_{+}^{\prime N} \mid S_{-}^{\prime \prime}}-\left.\mu\right|_{S_{+}^{\prime N}}\right\|_{t v}$ is a random variable, since it depends on the scenery on $S_{-}^{\prime \prime}$.) With $\left(X_{n}^{\prime}\right)_{n>0}$ and $\left(X_{n}^{\prime \prime}\right)_{n \leq 0}$ fixed, call the set of sceneries on $S_{-}^{\prime \prime}$ for which the last limit is zero "good". Then this set of good sceneries has measure one. Now, if the scenery on $S_{-}^{\prime \prime}$ is not good, then we again let the second coordinate of $Y_{n}^{\prime \prime}$ for $n>0$ be the fixed element $f_{0} \in F$. On the other hand, if the scenery on $S_{-}^{\prime \prime}$ is good, then, by Goldstein [6] Theorem 2.1, there exists a coupling of $\left.\mu\right|_{S_{+}^{\prime} \mid S_{-}^{\prime \prime}}$ and $\left.\mu\right|_{S_{+}^{\prime}}$ under which the two processes are successfully coupled after some random time.

Let $\nu$ denote the latter coupling (which depends on $\left(X_{n}^{\prime}\right)_{n>0},\left(X_{n}^{\prime \prime}\right)_{n \leq 0}$ and the scenery on $\left.S_{-}^{\prime \prime}\right)$. Let the second coordinate of $Y_{n}^{\prime \prime}$ for $n>0$ be given by the first marginal of $\nu$ conditioned on the second marginal of $\nu$ being the scenery $C^{\prime}$ on $S_{+}^{\prime}=S_{+}^{\prime \prime}$. This completes the definition of $Y^{\prime}$ and $Y^{\prime \prime}$, and from the construction it follows (after some reflection) that conditions (1), (2) and (3) hold.

STEP 2. (§) holds when $\Phi_{\mu}<\infty$ a.s.

Proof. The fact that $\Phi_{\mu}<\infty$ a.s. plays a crucial role, for one, because it implies that $|Z|<\infty$ a.s. (recall Remark (b) below Theorem 3). Consequently, the sets $S_{-}$and $S_{+}^{N}$ a.s. move apart as $N \rightarrow \infty$, which is of course vital for ( $\left.\S\right)$. The argument below is inspired by Burton and Steif [3] Section 2.

Fix $S$ for which $\Phi_{\mu}<\infty$. Choose $N$ so large that $S_{-}$and $S_{+}^{N}$ are disjoint, and define

$$
\delta_{N}=\sum_{\substack{x \in S_{+}^{N} \\ y \in S_{-}}} \phi_{\mu}(x-y) .
$$

Fix finite sets $A$ and $B$ such that $A \subseteq S_{+}^{N}$ and $B \subseteq S_{-}$. Let

$$
\begin{aligned}
& E_{\neq}(A, B)=\left\{\left(c^{\prime}, c^{\prime \prime}\right):\right. \exists x \in A, y \in B, \\
&\left.\pi: x \leftrightarrow y \text { such that } c^{\prime}(z) \neq c^{\prime \prime}(z) \forall z \in \pi\right\} .
\end{aligned}
$$

We have

$$
\begin{aligned}
(\mu \times \mu)\left(E_{\neq}(A, B)\right) & \leq \sum_{\substack{x \in A \\
y \in B}}(\mu \times \mu)\left(E_{\neq}(x, y)\right) \\
& \leq \sum_{\substack{x \in S_{+}^{N} \\
y \in S_{-}}} \phi_{\mu}(x-y)=\delta_{N}
\end{aligned}
$$

Let

$$
K=\left\{c \in F^{B}:\left(\mu^{c} \times \mu\right)\left(E_{\neq}(A, B)\right) \leq \sqrt{\delta_{N}}\right\}
$$


where $\mu^{c}$ denotes the conditional measure given that the scenery on $B$ is $c$. Below we will prove that

$$
\left\|\left.\mu^{c}\right|_{A}-\left.\mu\right|_{A}\right\|_{t v} \leq 2 \sqrt{\delta_{N}} \quad \forall c \in K
$$

Next, continuing from $(\dagger)$, we have

$$
\delta_{N} \geq\left.\int_{c \in F^{B} \backslash K} \mu\right|_{B}(d c)\left(\mu^{c} \times \mu\right)\left(E_{\neq}(A, B)\right) \geq \sqrt{\delta_{N}}\left[1-\left.\mu\right|_{B}(K)\right],
$$

and so

$$
1-\left.\mu\right|_{B}(K) \leq \sqrt{\delta_{N}}
$$

It follows that

$$
\begin{aligned}
& \left\|\left.\mu\right|_{A \cup B}-\left.\mu\right|_{A} \times\left.\mu\right|_{B}\right\|_{t v} \\
& \quad \leq\left.\int_{c \in F^{B}} \mu\right|_{B}(d c)\left\|\left.\mu^{c}\right|_{A}-\left.\mu\right|_{A}\right\|_{t v} \\
& \quad \leq\left. 2 \sqrt{\delta_{N}} \mu\right|_{B}(K)+2\left[1-\left.\mu\right|_{B}(K)\right] \leq 4 \sqrt{\delta_{N}},
\end{aligned}
$$

where the second inequality uses $(\dagger)$. Taking the supremum over $A$ and $B$, we get

$$
\begin{aligned}
&\left\|\left.\mu\right|_{S_{-} \cup S_{+}^{N}}-\left.\mu\right|_{S_{-}} \times\left.\mu\right|_{S_{+}^{N}}\right\|_{t v} \\
& \quad=\sup _{\substack{A \subseteq S_{+}^{N} \\
|A|<\infty}} \sup _{\substack{B \subseteq S_{-} \\
|B|<\infty}}\left\|\left.\mu\right|_{A \cup B}-\left.\mu\right|_{A} \times\left.\mu\right|_{B}\right\|_{t v} \leq 4 \sqrt{\delta_{N}} .
\end{aligned}
$$

Finally, we let $N \rightarrow \infty$ and use that $\lim _{N \rightarrow \infty} \delta_{N}=0$ because $\Phi_{\mu}<\infty$, to obtain $(\S)$.

It remains to prove $(\dagger)$. We show that, for any $c \in K$, the measures $\mu^{c}$ and $\mu$ can be successfully coupled on $A$ with a probability at least $1-\sqrt{\delta_{N}}$. From this, $(\dagger)$ follows via the basic coupling inequality relating total variation norms to couplings (see Lindvall [10] Section I.2).

Fix $c \in K$. Let $\left(\sigma_{1}, \sigma_{2}\right)$ be chosen according to $\mu^{c} \times \mu$. Let $U$ be the set of sites that can be reached from $B$ via a path of disagreement in $\left(\sigma_{1}, \sigma_{2}\right)$. Our assumption that $\sum_{x} \phi_{\mu}(x)<\infty$ implies that $\left(\mu^{c} \times \mu\right)(|U|<\infty)=1$. Next, define $\left(\eta_{1}, \eta_{2}\right)$ as follows:

$$
\begin{aligned}
\left(\eta_{1}, \eta_{2}\right)=\left(\sigma_{1}, \sigma_{2}\right) & \text { if } U \cap A \neq \emptyset \\
& =\left(\sigma_{1}, \hat{\sigma}_{2}\right) \text { if } U \cap A=\emptyset
\end{aligned}
$$


where

$$
\begin{aligned}
\hat{\sigma}_{2}(x) & =\sigma_{2}(x) \quad \text { for } x \in U, \\
& =\sigma_{1}(x) \text { for } x \in U^{c} .
\end{aligned}
$$

The Markov property of $\mu$ implies that $\eta_{2}$ has distribution $\mu$ (while, of course, $\eta_{1}$ has distribution $\mu^{c}$ ). By construction, if $\eta_{1}$ and $\eta_{2}$ differ somewhere on $A$, then there must be a path of disagreement from $B$ to $A$ in $\left(\eta_{1}, \eta_{2}\right)$. Hence $\eta_{1}$ and $\eta_{2}$ differ somewhere on $A$ with probability at most $\left(\mu^{c} \times \mu\right)\left(E_{\neq}(A, B)\right)$, which is $\leq \sqrt{\delta_{N}}$ because $c \in K$.

\section{Proof of Theorem 4}

We will prove that $\tilde{\Phi}_{\mu}<\infty$ a.s. implies property $(\S)$ introduced at the beginning of Section 4. The weak Bernoullicity of $Y$ will then follow from Step 1 in Section 4 . We will in fact prove the stronger property that

$$
\lim _{N \rightarrow \infty} \sup _{\substack{A \subseteq S_{+}^{N} \\|A|<\infty}} \sup _{\substack{B \subseteq S_{-} \\|B|<\infty}} \sup _{\eta \in F^{B}}\left\|\left.\mu\right|_{A \mid \eta}-\left.\mu\right|_{A}\right\|_{t v}=0
$$

for any $S$ for which $\tilde{\Phi}_{\mu}<\infty$.

Fix $\epsilon>0$ and $S$ for which $\tilde{\Phi}_{\mu}<\infty$. Pick $N$ large enough so that

$$
\sum_{\substack{x \in S_{+}^{N} \\ y \in S_{-}}} P_{p(\mu)}(x \longleftrightarrow y)<\epsilon / 2
$$

Let $A \subseteq S_{+}^{N}, B \subseteq S_{-}$and $\eta \in F^{B}$, with $A, B$ finite. Then $P_{p(\mu)}(A \longleftrightarrow B)<\epsilon / 2$. Next, choose $k$ so large that $A \cup B \subseteq B_{k}=[-k, k]^{d} \cap \mathbb{Z}^{d}$ and $P_{p(\mu)}\left(A \longleftrightarrow B_{k}^{c}\right)<$ $\epsilon / 2$, the latter being possible because $\sum_{x} \tilde{\phi}_{\mu}(x)<\infty$. Put $C=B_{k} \backslash B$. Then $P_{p(\mu)}\left(A \longleftrightarrow C^{c}\right)<\epsilon$ because $C^{c}=B \cup B_{k}^{c}$.

Next, write

$$
\left\|\left.\mu\right|_{A \mid \eta}-\left.\mu\right|_{A}\right\|_{t v} \leq\left.\int_{\sigma \in F^{B_{k}^{c}}} \mu\right|_{B_{k}^{c}}(d \sigma)\left\|\left.\mu\right|_{A \mid(\sigma, \eta)}-\mu_{A}\right\|_{t v} .
$$

The key step in the argument now is van den Berg and Maes [2] Corollary 1, which implies that

$$
\left\|\left.\mu\right|_{A \mid(\sigma, \eta)}-\left.\mu\right|_{A}\right\|_{t v} \leq P_{p(\mu)}\left(A \longleftrightarrow C^{c}\right) \quad \forall \sigma \in F^{B_{k}^{c}}, \quad \eta \in F^{B} .
$$

The right-hand side is $<\epsilon$. Take the supremum over $A, B$ and let $\epsilon \downarrow 0$ to complete the proof. 


\section{Proof of Theorem 5}

Given $m$, let $\tilde{m}$ be defined by $\tilde{m}(x)=\frac{1}{2} \delta_{x 0}+\frac{1}{2} m(x), x \in \mathbb{Z}^{d}$. In other words, we make the random walk pause with probability $\frac{1}{2}$. In doing so, we obtain a random walk that is "genuinely $d$-dimensional" (recall that $m$ is irreducible) in the sense of Spitzer [12] Proposition 7.6, which will be needed in part (i) below. The pausing leaves $S_{-}, S_{+}$invariant, and hence also $F$.

Let $P, E$ denote probability and expectation with respect to $\tilde{S}$, the random walk associated with $\tilde{m}$. Then

$$
\begin{aligned}
E(F) & =\sum_{x, y \in \mathbb{Z}^{d}} f(x-y) P\left(y \in \tilde{S}_{-}\right) P\left(x \in \tilde{S}_{+}\right) \\
& \leq \sum_{x, y \in \mathbb{Z}^{d}} f(x-y) \sum_{m \geq 0} p_{m}(y, 0) \sum_{n>0} p_{n}(0, x)
\end{aligned}
$$

where $p_{n}(x, y)$ is the $n$-step transition probability between $x$ and $y$ for $\tilde{S}$. Write $p_{m}(y, 0) p_{n}(0, x)=p_{m}(0,-y) p_{n}(-y, x-y)$, sum over $y$ while keeping $z=x-y$ fixed, and use the identity $\sum_{y} p_{m}(0,-y) p_{n}(-y, z)=p_{m+n}(0, z)$, to obtain

$$
E(F) \leq \sum_{z \in \mathbb{Z}^{d}} f(z) \sum_{k>0} k p_{k}(0, z)
$$

(i) According to Spitzer [12] Proposition 7.6, we have

$$
p_{k}(0, z) \leq C k^{-d / 2} \text { for } k>0, z \in \mathbb{Z}^{d} \text { and some } C<\infty .
$$

Thus, if $d \geq 5$, then the sum over $k$ is bounded uniformly in $z$, and so $E(F)<\infty$ by our assumption that $\sum_{x} f(x)<\infty$.

(ii) Let $\sum_{x} x \tilde{m}(x)=v \neq 0$. Pick $0<\epsilon<\|v\|$. For fixed $k$, split the sum over $z$ into two parts:

$$
I_{k}=\sum_{z:\|z-v k\| \leq \epsilon k} f(z) k p_{k}(0, z) \text { and } I I_{k}=\sum_{z:\|z-v k\|>\epsilon k} f(z) k p_{k}(0, z) .
$$

In $I_{k}$, use that $\|z\| \geq(\|v\|-\epsilon) k$, to estimate

$$
\begin{aligned}
\sum_{k>0} I_{k} & \leq \frac{1}{\|v\|-\epsilon} \sum_{z \in \mathbb{Z}^{d}}\|z\| f(z) \sum_{k>0} p_{k}(0, z) \\
& \leq \frac{1}{\|v\|-\epsilon} \sum_{z \in \mathbb{Z}^{d}}\|z\| f(z) \sum_{k \geq 0} p_{k}(0,0) .
\end{aligned}
$$


The sum over $k$ is finite because the random walk is transient by our assumption that $v \neq 0$. The sum over $z$ is finite by our assumption that $\sum_{x}\|x\| f(x)<\infty$. In $I I_{k}$, on the other hand, use that $f \leq 1$, to estimate

$$
\begin{aligned}
\sum_{k>0} I I_{k} & \leq \sum_{k>0} k P\left(\left\|\tilde{S}_{k}-E\left(\tilde{S}_{k}\right)\right\|>\epsilon k\right) \\
& \leq \frac{1}{\epsilon^{6}} \sum_{k>0} \frac{1}{k^{5}} E\left(\left\|\tilde{S}_{k}-E\left(\tilde{S}_{k}\right)\right\|^{6}\right) .
\end{aligned}
$$

Since $\tilde{S}_{k}=\sum_{l=1}^{k} \tilde{X}_{l}$, where the increments are independent with common distribution $\tilde{m}$, it is not too difficult to invoke our assumption that $\sum_{x}\|x\|^{6} \tilde{m}(x)<\infty$ to deduce that

$$
\begin{array}{r}
E\left(\left\|\tilde{S}_{k}-E\left(\tilde{S}_{k}\right)\right\|^{6}\right) \leq d^{6} \sum_{i=1}^{d} E\left(\left[\sum_{l=1}^{k}\left(X_{l}^{i}-E\left(X_{l}^{i}\right)\right)\right]^{6}\right) \\
\text { for } k>0 \text { and some } C<\infty .
\end{array}
$$

Hence the last sum is also finite.

\section{References}

[1] H.C.P. Berbee, Random Walks with Stationary Increments and Renewal Theory, Math. Centre Tracts 112, Centre for Mathematics and Computer Science, Amsterdam, 1979.

[2] J. van den Berg and C. Maes, Disagreement percolation in the study of Markov fields, Ann. Probab., 22 (1994), 749-763.

[3] R.M. Burton and J.E. Steif, Quite weak Bernoulli with exponential rate and percolation for random fields, Stochastic Process. Appl., 58 (1995), 35-55.

[4] H.-O. Georgii, Gibbs Measures and Phase Transitions, de Gruyter, Berlin, 1988.

[5] H.-O. Georgii, Mixing properties of induced random transformations, Erg. Th. Dynam. Syst., 17 (1997), 839-847.

[6] S. Goldstein, Maximal coupling, Z. Wahrsch. Verw. Gebiete, 46 (1979), 193-204.

[7] G. Grimmett, Percolation (2nd. ed.), Springer, Berlin, 1999.

[8] F. den Hollander and J.E. Steif, Mixing properties of the generalized $T, T^{-1}$-process, J. Analyse Math., 72 (1997), 165-202.

[9] G. Lawler, Intersections of Random Walks, Birkhäuser, Boston, 1991.

[10] T. Lindvall, Notes on the Coupling Method, Wiley, New York, 1992.

[11] I. Meilijson, Mixing properties of a class of skew-products, Israel J. Math., 19 (1974), 266270.

[12] F. Spitzer, Principles of Random Walk (2nd ed.), Springer, New York, 1976.

[13] P. Walters, An Introduction to Ergodic Theory, Springer, New York, 1975. 
F. DEN HOLLANDER

EURANDOM, P.O. BOX 513

5600 MB Eindhoven, The NetherLands

E-mail: denhollander@eurandom.tue.nl

\section{M.S. KeANe}

Mathematics Department WESLEYAN UNIVERSITY

MidDLETOWn, CT 06459-0128, USA

E-mail: mkeane@wesleyan.edu AND

Korteweg-de VRies Institute

UNIVERSITY OF AMSTERDAM

Amsterdam, The Netherlands

\section{J. Serafin}

INSTITUTE OF Mathematics

WROCEAW UNIVERSITY OF TECHNOLOGY

W YBRZEŻE WYSPIAŃSKIEGO 27

50-370 Wroceaw, Poland

E-mail: serafin@im.pwr.wroc.pl

J.E. STEIF

Department of Mathematics Chalmers University of Technology

S-41296 Gothenburg, Sweden

E-mail: steif@math.chalmers.se AND

School of Mathematics

Georgia Institute of Technology Atlanta, GA 30332-0160, USA 